\title{
Wicked Problems und Digitalisierung als Herausforderung für politisch-administra- tives Handeln
}

\author{
Olaf Winkel
}

Als Wicked Problems werden gesellschaftliche Herausforderungen bezeichnet, die sich herkömmlichen Bearbeitungsstrategien von Politik, Verwaltung und anderen mit der Erfüllung öffentlicher Aufgaben befassten Akteuren entziehen. Im Übergang zur digitalen Informationsgesellschaft ist mit der Entstehung neuer und der Verschärfung überkommener Wicked Problems zu rechnen, weil die Verlagerung zentraler gesellschaftlicher Funktionen in elektronische Netzwerke einer Erhöhung sozialer Komplexität, Diversität und Unsicherheit Vorschub leistet und dazu beiträgt, dass Zielkonflikte auf die Spitze getrieben werden. Zur Anpassung der gesellschaftlichen Problembearbeitungsfähigkeit an die gestiegenen Anforderungen sind kulturelle und institutionelle Innovationen erforderlich.

\section{Einführung}

Wicked Problems stellen Politik und Verwaltung vor große Herausforderungen. Der vorliegende Beitrag soll zeigen, dass und aus welchen Gründen der Übergang zur digitalen Informationsgesellschaft der Entstehung neuer bzw. der Verschärfung überkommener Wicked Problems Vorschub leistet, wobei ausgewählte Handlungsfelder exemplarische Beachtung finden. Grundlage der Untersuchung ist die Aufarbeitung von Phänomenen und Entwicklungen, die mit den Begriffen Wicked

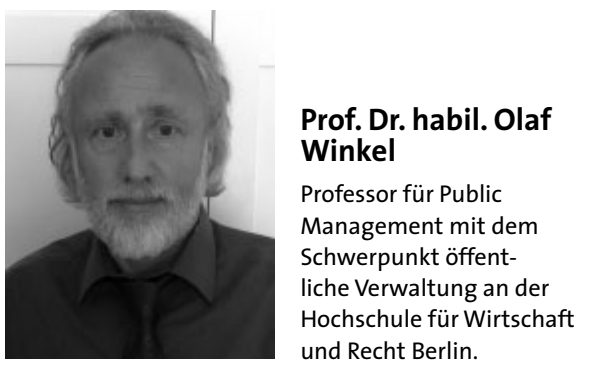

Problems und Digitalisierung verbunden werden.

\section{Wicked Problems}

Wicked Problems finden sich in den Ländern der westlichen Welt unter anderem dort, wo Fragen des technischen Fortschritts, des Klimawandels, der ökonomischen Wohlfahrt, der sozialen Segmentierung, der demographischen Entwicklung oder des internationalen Terrorismus virulent werden. ${ }^{1}$

Das Konzept der Wicked Problems geht auf planungstheoretische Überlegungen zurück, die Horst Rittel in den sechziger Jahren des 20. Jahrhunderts in Lehrveranstaltungen formulierte und die von ihm erst später einer breiteren Fachöffentlichkeit zugänglich gemacht wurden. ${ }^{2}$ Eine erste Publikation zu diesem Thema stammt daher nicht von Rittel, sondern von Charles Churchman, der den Kern von dessen Aussagen treffend auf den
Punkt bringt. ${ }^{3}$ Das Adjektiv „wicked“, also „bösartig“, stellt dabei keine moralisierende Wertung dar, sondern bezieht sich auf die besonderen Schwierigkeiten, die den Verantwortlichen bei der Bearbeitung von Wicked Problems erwachsen, so dass hier auch von „vertrackten“ oder „verzwickten“ Problemen gesprochen werden kann. ${ }^{4}$

Zur Kennzeichnung solcher Probleme und den mit ihrer Bearbeitung verbundenen Herausforderungen führen Rittel und Webber folgende Merkmale auf ${ }^{5}$ :

Wicked Problems lassen sich nicht eindeutig beschreiben. Dies ist mit Faktoren wie unklaren Ursache-Wirkungs-Beziehungen, der Verzahnung von Problembeschreibung und antizipierter Problembearbeitung und der Vielfalt möglicher Sichtweisen im Zusammenhang zu sehen.

Ein Wicked Problem hat „keine Stoppregel“ ${ }^{\text {, }}$, d.h. schon weil es sich nicht eindeutig definieren lässt, liefert es keine immanenten Hinweise darauf, wann die Bemühungen zu seiner Bearbeitung eingestellt werden können. Lösungen für Wicked Problems sind nicht richtig oder falsch, sondern gut oder schlecht. Weil objektive Beurteilungskriterien fehlen,

\footnotetext{
Siehe etwa Fischbacher-Smith 2016, Hulme 2009 Levin/Cashore/Bernstein/Graeme 2012, Ritchey 2013, S. 2, Spicker 2016.

Siehe Rittel/Webber 1973, S. $155 \mathrm{ff}$

Siehe Churchman 1967, S. $141 f$.

Rittel/Webber 1973, S. 160.

Ebenda, S. 161ff
} 
können Lösungsansätze und ihre Wirkungen von unterschiedlichen Seiten in unterschiedlicher Weise wahrgenommen und bewertet werden.

Ob bzw. inwieweit ein Wicked Problem überwunden ist, lässt sich nicht überprüfen. Dies ist unter anderem darauf zurückzuführen, dass die gewählten Maßnahmen nicht nur unerwünschte Nebenwirkungen,
Die Art der Problemerklärung und die Wahl der Problembearbeitungsstrategie bedingen sich wechselseitig. Weil Beteiligte und Betroffene Wicked Problems unterschiedlich wahrnehmen und damit unterschiedliche Wertvorstellungen und Interessen verbinden, können hier diametral entgegengesetzte und dennoch aus der jeweiligen Einzelperspektive durchaus legitime Positionen aufeinandertreffen.

\section{"Als Wicked Problems werden gesellschaftliche Herausforderungen bezeichnet, die sich überkommenen Bearbeitungsstrategien von Politik und Verwaltung entziehen."}

sondern auch noch viel später schädliche Nachwirkungen zeitigen können, die gegenüber den angezielten Effekten überwiegen.

Jede Lösung eines Wicked Problems ist ein nicht rückgängig zu machender Vorgang. Daher kommt hier Lernen nach dem Prinzip von Versuch und Irrtum nicht in Betracht.

Zur Bearbeitung von Wicked Problems ist jeweils eine Unzahl möglicher Konzepte denkbar, wobei die Präferenz für eine Lösungsstrategie maßgeblich von dem Bild abhängt, das sich Betrachter von der Art des Problems und von wünschenswerten Entwicklungsperspektiven machen. Ein Wicked Problem ist einzigartig, so dass sich die Übertragung von Lösungsansätzen von einem Problemfeld auf ein anderes verbietet.

Jedes Wicked Problem kann als Symptom eines anderen betrachtet werden. Die zahlreichen Wechselwirkungen zwischen relevanten Faktoren innerhalb und außerhalb des Problemfelds implizieren auch, dass das Problem eine Vielzahl von in unterschiedlichen Bereichen und auf unterschiedlichen Ebenen angesiedelten Entscheidungsträgern adressiert.
Weil Lebenswelten durch entsprechende Maßnahmen irreversibel verändert werden, haben die für die Bearbeitung von Wicked Problems Verantwortlichen kein Recht auf Irrtum.

Die aufgeführten Merkmale implizieren auch, dass sich Wicked Problems nicht umfassend lösen, sondern lediglich partiell und temporär entschärfen lassen. Dies bringt Churchman in der Metapher, dass man zwar das „Knurren der Bestie“ besänftigen, aber niemals die Bestie selbst zähmen könne, anschaulich zum Ausdruck. ${ }^{7}$ Als Triebkräfte bzw. als „typische Eigenschaften“ von Wicked Problems gelten „Komplexität, Diversität und Unsicherheit“. ${ }^{8}$

Als komplex kann man soziale Konstellationen bezeichnen, die durch eine Vielfalt von unterschiedlich ausgeprägten und in engen Wechselwirkungen stehenden Faktoren bestimmt werden. ${ }^{9}$

Diversität seht für soziale Vielfalt, wobei Alter, ethnische Herkunft, sexuelle Identität, weltanschauliche Präferenzen, Religionszugehörigkeit, Lebensstil, familiäre Situation und ökonomischer Status zu den zentralen Dimensionen von Diversität zählen. ${ }^{10}$ Steigende Diversität impliziert auch zunehmende Divergenz und
Fragmentierung hinsichtlich der Werthaltungen, Einstellungen und Interessen der Gesellschaftsmitglieder. ${ }^{11}$ Viele sehen hier den Kern von Wicked Problems, was auch darin zum Ausdruck kommt, dass gelegentlich alternativ von „Divergent Problems " die Rede ist. ${ }^{12}$

Als unsicher gelten soziale Situation, in denen Individuen außerstande sind, ein verlässliches Bild von den zukünftigen Auswirkungen eigener und fremder Handlungen zu gewinnen. ${ }^{13}$ Verwandte Begriffe sind Ungewissheit, Risiko und Unwissen.

Wenn Wicked Problems zur Bearbeitung anstehen, stoßen überkommene lineare Aktivitäten und Routinen von Politik und Verwaltung an ihre Grenzen. ${ }^{14}$ Für den Umgang mit Problemen dieser Art werden den Verantwortlichen unter anderem Ehrlichkeit bei ihrer Beurteilung, Denken in Systemen, eine behutsame Herangehensweise, Kreativität und Offenheit bei der Lösungssuche, ein inkrementalistisches, aber gleichzeitig Gesamtzusammenhänge nicht vernachlässigendes Vorgehen und der Einsatz unterschiedlicher Instrumente zur Unterstützung von Planungsund Entscheidungsprozessen empfohlen. ${ }^{15}$

Als zentraler strategischer Ansatzpunkt zur Entschärfung von Wicked Problems gilt eine breit angelegte Kollaboration, die Beteiligte und Betroffene mit ihren unterschiedlichen Wahrnehmungen, Wertvorstellungen und konkreten Interessen so weit wie möglich in Diskurse und Entscheidungsprozesse einbezieht. ${ }^{16}$

6 Ebenda, S. 162.

Churchman 1967, S. 141.

$8 \mathrm{Head} /$ Alford 2015, S. 716.

9 Siehe Fuchs-Heinritz/Lautmann/Rammstedt/ Wienold 1995, S. 354.

10 Siehe Schwarz-Wölzl/Maad 2004, S. $4 \mathrm{ff}$.

1 Siehe Head 2008, S. 103.

12 Schumacher 1995.

13 Siehe Banse 1996, S. $15 \mathrm{ff}$.

14 Siehe etwa Head/Alford 2015, S. 712f., Levin/ Cashore/Bernstein/Graeme 2012, S. 123ff., Watkins/Wilber 2015, S. $53 \mathrm{ff}$.

15 Siehe etwa AGPSC 2007, S. 35ff., Churchman 1967, S. 142, Horn/Weber 2007.

16 Siehe etwa Goldsmith/Eggers 2004, Head 2008, S. 114f., Weber/Khademian 2008, S. 334ff. 
Dies unterstreicht die Bedeutung von Governance-Netzwerken, in denen Akteure aus den unterschiedlichsten gesellschaftlichen Bereichen mit Akteuren aus Politik und Verwaltung zusammenwirken. Wissenschaftlichen Einrichtungen werden dabei Funktionen zugeordnet, die das „traditionelle Wissenschaftsmodell hinter sich lassen "17, nach dem sich deren Rolle weitgehend in der Gewinnung und Verbreitung relevanter Erkenntnisse erschöpft. Sie sollen auch als Frühwarnsysteme dienen und sich als „Makler und Mediatoren “ in die Netzwerke einfügen. ${ }^{18}$

\section{Digitalisierung}

Als Digitalisierung bezeichnet man das Umwandeln von analogen Daten in ein Format, das einer maschinellen Bearbeitung besser zugänglich ist. Dabei werden große Datenmengen von ihren speziellen Trägern gelöst und in elektronische Netzwerke verlagert. ${ }^{19}$ In dem Maße, wie dieser Prozess und die „Verknüpfung der virtuellen mit der realen Welt" voranschreiten $^{20}$, werden die Merkmale des virtuellen Raums zu Merkmalen der Gesellschaft selbst.

Dabei ist zu berücksichtigen, dass die Digitalisierung Entwicklungen Vorschub leistet, wie man sie mit den Begriffen Globalisierung, Ausdifferenzierung und Beschleunigung verbindet.

Als Globalisierung bezeichnet man Prozesse, in deren Verlauf immer mehr Akteure aus Wirtschaft und Gesellschaft unabhängig von ihren nationalstaatlichen Repräsentanten in grenzüberschreitende Kooperationsbeziehungen eintreten. ${ }^{21}$ Weil Informationen, die irgendwo in digitale Netzwerke eingespeist werden, prinzipiell weltweit jeder Person mit Internetzugang zur Verfügung stehen, begünstigt der Übergang vom Analogen zum Digitalen Globalisierungsschübe.

Soziale Ausdifferenzierung, zu verstehen als „Aufgliederung eines gesellschaftlichen Ganzen“22, manifestiert sich in der Zunahme von Subkulturen und der Herausbildung immer bunterer Lebenswelten. Das Ausmaß der Ausdifferenzierung hängt dabei stark von den zur Verfügung stehenden Interaktionsmöglichkeiten ab. Sind sie gering, werden Differenzierungspro-

zesse eingedämmt und Lebenswelten uniform gehalten, sind sie umfassend, werden Differenzierungsprozesse gefördert und Lebenswelten vielfältiger. Grenzüberschreitend angelegte elektronische Netzwerke, die neue Räume schaffen, in denen Gleichgesinnte zueinander finden, sich austauschen und auch eine über die virtuelle Welt hinausreichende Anschlusskommunikation organisieren können, zählen daher zu den zentralen Triebkräften der gesellschaftlichen Ausdifferenzierung.

\section{"Digitalisierung steht für die Umwandlung analoger Daten in ein maschinenlesbares Format. Dabei werden große Datenmengen von ihren Trägern gelöst und in elektronische Netzwerke verlagert. In dem Maße, wie dieser Prozess voranschreitet, werden die Merkmale des virtuellen Raums zu Merkmalen der Gesellschaft selbst."}

Beschleunigung steht aus einer soziologischen Perspektive nicht nur für den schnelleren Ablauf von Kommunikations- und Kooperationsprozessen, sondern ebenso für die Erhöhung der Geschwindigkeit, in der sich Innovationen vollziehen. ${ }^{23}$ Dies bedeutet auch, dass sich die Gesellschaftsmitglieder ,zunehmend schneller an neue Lebensformen, Handlungsbedingungen und Situationsbestimmungen anpassen " müssen ${ }^{24}$, und dass wirtschaftliche Entwicklungen einschließlich der Herausbildung neuer Arbeitsteilungsmuster, Allokationsformen und Geschäftsmodelle eine bislang nicht gekannte Dynamik entfalten. Dabei kann die „Beschleunigung des sozialen Wandels“ als „direkte Folge der technischen Beschleunigung “ interpretiert werden. ${ }^{25}$

Neben den geschilderten Tendenzen zu Globalisierung, Ausdifferenzierung und Beschleunigung ist zum Verständnis der gesellschaftlichen Auswirkungen der Digitalisierung zu beachten, dass die Funktionalitäten elektronischer Netzwerke weniger von der Hardware als von der Software bestimmt werden, die keiner fixen technischen, sondern einer disponiblen sozialen Logik folgt. Nach dem eine Suchmaschine prinzipiell auch so konzipiert werden, dass die Kommunikationsakte und Kommunikationsinhalte der Nutzer vertraulich bleiben.

Die Bereiche, in denen Politik und Verwaltung in Ländern westlicher Prägung im Übergang zur digitalen Informationsgesellschaft aktiv werden müssen, sind zahlreich. $^{27} \mathrm{Zu}$ den zentralen Herausforderungen, die unmittelbar mit dieser Entwicklung zusammenhängen, zählen etwa der Ausbau der digitaltechnischen Infrastruktur, die Förderung von Medienkompetenz, die Unterstützung innovativer Wirtschaftsunternehmen, die Schaffung

\footnotetext{
17 Weber/Lach/Steel 2017, S. 9.

18 Brugnach/Ingram 2017, S. 38.

19 Siehe etwa Bleicher 2010, Wolf/Strohchen 2018.

20 Hirsch-Kreinsen 2016, S. 10.

Siehe etwa Beck 1998.

22 Fuchs-Heinritz/Lautmann/Rammstedt/Wienold 1995, S. 14.

3 Siehe etwa Rosa 2005

24 Cheng 2012, S. 45.

Rosa 2005 , S. 248.

26 Lessig 1997

27 Siehe etwa Martini 2016, OECD 2019, S. 15ff., Winkel 2018, S. 121ff.
} 
von Rahmenbedingungen für eine sozialverträgliche Transformation des Erwerbslebens, die Förderung von Orientierung als Gegengewicht zur Informationsüberflutung, die Bewahrung einer politischen Öffentlichkeit, die Aufrechterhaltung der öffentlichen Sicherheit und die Verteidigung von Privatheit.

\section{Wicked Problems und Digital- isierung in der Zusammensicht}

Dass Wicked Problems im Übergang zur digitalen Informationsgesellschaft auf dem Vormarsch sind, ist vor allem auf zwei Gründe zurückzuführen. Die erste besteht darin, dass die Digitalisierung in Verbindung mit den Tendenzen zur Globalisierung, Ausdifferenzierung und Beschleunigung einer Erhöhung von Komplexität, Diversität und Unsicherheit Vorschub leistet. Der zweite resultiert daraus, dass die zunehmende Gestaltung sozialer Beziehungen durch Software eine Verschärfung von Zielkonflikten mit sich bringt.

\section{Erhöhung von Komplexität, Diversität und Unsicherheit}

Die ineinander verwobenen Entwicklungen, die mit den Begriffen Digitalisierung, Globalisierung, Ausdifferenzierung und Beschleunigung assoziiert werden, wirken komplexitätssteigernd, weil sie die Anzahl von Parametern und Wechselwirkungen in sozialen Konstellationen erhöhen und das Tempo steigern, in dem diese entstehen und sich verändern. Als Diversität erhöhend sind diese Entwicklungen anzusehen, weil ein Anwachsen sozialer Vielfalt durch eine mit zunehmender Geschwindigkeit erfolgende Ausbildung von Subkulturen ihren Niederschlag auch darin findet, dass die Werthaltungen, Einstellungen und Interessen der Gesellschaftsmitglieder immer weiter auseinanderklaffen. Dies wird besonders deutlich, wenn man sich vergegenwärtigt, dass die Begriffe Ausdifferenzierung und Diversität letztlich auf identische Phänomene verweisen. Während der erstgenannte für den Prozess des Auseinanderfallens ehemals geteilter Überzeugungen steht, steht der letztgenannte für das Ergebnis dieses Prozesses.

Dass die Unsicherheit mit zunehmender Digitalisierung, Globalisierung,
Ausdifferenzierung und Beschleunigung wächst, ist schon deshalb evident, weil dies eine unvermeidliche Folge des technisch induzierten Anwachsens von Komplexität und Diversität darstellt. Immer komplexere Lebenswelten sind immer schwerer zu überschauen und in ihren Fortentwicklungen $\mathrm{zu}$ antizipieren. Hinzu kommt, dass den Gesellschaftsmitgliedern mit zunehmender Geschwindigkeit der Veränderungen immer weniger Zeit bleibt, diese zu reflektieren und sich auf sie einzustellen.

In nahezu allen Handlungsfeldern, die mit dem Übergang zur digitalen Infor- und der Übertragung von Rundfunksendungen dienen sollten. Bei der Planung, Herstellung und Nutzung der Infrastruktur dominierte ein nationaler Fokus.

Heute geht es hier darum, neben dem Festnetz auch den Mobilfunk auszubauen und beide Technologien in einer Weise zu verzahnen, die der Fortentwicklung virtueller Netze Vorschub leistet. Gemeint sind damit Netze wie das Internet, die sich nicht physikalisch, sondern über Softwarestandards und Protokolle konstituieren. Dabei ist der nationale Fokus bei der Planung, Herstellung und Nutzung der informationstechnischen Infrastruktur

\section{"Die ineinander verwobenen Phänomene der Digitalisierung, Globalisierung, Ausdifferenzierung und Beschleunigung begünstigen Wicked Problems, weil sie der Erhöhung von Komplexität, Diversität und Unsicherheit Vorschub leisten."}

mationsgesellschaft im Zusammenhang stehen, sind Komplexitätserhöhungen zu verzeichnen, zumeist begleitet von steigender Diversität und Unsicherheit. Dies gilt auch im Hinblick auf den Ausbau der informationstechnischen Infrastruktur und die Förderung von Medienkompetenz als zwei Bereiche, die im Folgenden exemplarisch angesprochen werden.

Tragfähige Infrastrukturen gelten als unabdingbare Voraussetzung für volkswirtschaftliche Leistungsfähigkeit und gesellschaftliche Wohlfahrt. Dabei wird der informationstechnischen Infrastruktur im Übergang zur digitalen Informationsgesellschaft sogar eine Schlüsselfunktion zugemessen.

Vor der Herausbildung der digitalen Netzkommunikation konzentrierte sich der Ausbau der informationstechnischen Infrastruktur auf die Schaffung vorwiegend hardwarebasierter Festnetze, die insbesondere als Basis des Telefondienstes längst einer Betrachtungsweise gewichen, in der internationale Aspekte eine zentrale Rolle spielen. So stellt sich gegenwärtig in europäischen Ländern die Frage, ob man dem chinesischen Unternehmen Huawei die Möglichkeit geben soll, an der Aufrüstung der europäischen Mobilkommunikation nach dem 5G-Standard mitzuwirken oder nicht. ${ }^{28}$ Und bei der Beurteilung dieser Frage sind nicht nur die Kostenvorteile einer solchen Lösung zu den Risiken in Bezug zu setzen, die aus der Nähe des Konzerns zur chinesischen Regierung erwachsen. Zu beachten sind auch Aspekte wie die gegenwärtigen Spannungen zwischen China und den USA und die Erwartungen, welche die Regierung in Washington an ihre europäischen Bündnispartner heranträgt.

28 Siehe etwa Siegele 2019, S. 23. 
Neu ist auch das Ausmaß, das die wechselseitige Abhängigkeit der unterschiedlichen infrastrukturellen Systeme angenommen hat. ${ }^{29}$ So sind das Verkehrswesen, das Gesundheitswesen, die öffentliche Verwaltung und das Finanzwesen heute auf eine zuverlässige informationstechnische Infrastruktur unabdingbar angewiesen, während das Funktionieren der informationstechnischen Infrastruktur wiederum eine zuverlässige Stromversorgung voraussetzt.

Vor nicht allzu langer Zeit galt Medienkompetenz vielen noch als „seelische Grundhaltung“, die Menschen befähigt, durch Massenmedien und insbesondere das Fernsehen übermittelte Inhalte als wertvoll oder minderwertig einzustufen und auf dieser Grundlage Informationen auszuwählen, die der eigenen Entwicklung förderlich sind. ${ }^{30}$ Mit dem Einzug des Computers in die Arbeitswelt und in immer mehr Bereiche des Privatlebens gewann dann eine Sichtweise an Bedeutung, die Medienkompetenz vorwiegend als „Informationstechnikkompetenz" versteht $^{31}$, d.h. als das Vermögen, informationstechnische Instrumente zielorientiert einzusetzen und die mit ihrer Nutzung verbundenen Vorteile und Risiken zu beurteilen.

Heute dominiert dagegen eine Lesart, die diesen Begriff wesentlich weiter auslegt. So ist etwa die Rede von Medienkompetenz als „Schlüssel zu einer Welt im Wandel“"32, d.h. als konstituierendes Element einer umfassenden Lebenskompetenz, die sich beschreiben lässt als Fähigkeit, in einer zunehmend digitalisierten und von vielen anderen ineinander verwobenen Veränderungsprozessen geprägten Welt im persönlichen Bereich, im Arbeitsleben und als Mitglied des politischen Gemeinwesens selbstbestimmt und sozial verantwortlich zu agieren. Umso breiter ihr Gegenstandsbereich ausgelegt wird, desto komplexer, vielschichtiger und bedeutsamer erscheint das mit der Förderung von Medienkompetenz verbundene Aufgabenspektrum.

\section{Zuspitzung von Zielkonflikten}

Wie eingangs dargelegt lassen sich gesellschaftliche Verhältnisse über die Gestaltung von Algorithmen weitaus effektiver beeinflussen als mit überkommenen Verfahren von Regelsetzung, Überprüfung der Regeltreue und Sanktionierung abweichenden Verhaltens. Wenn ein Ziel, das zu einem anderen in Konkurrenz steht, quasi in die Software hineingeschrieben und damit weitaus effektiver als in der Vergangenheit verfolgt werden kann, bedeutet dies aber natürlich auch, dass im Hinblick auf das andere Ziel erhebliche Rückschritte hingenommen werden müssen. Daher geht mit dem Übergang vom Analogen zum Digitalen eine Tendenz zur Verschärfung von Zielkonflikten einher. ${ }^{33}$ isch ausgeleuchtete Konflikt zwischen Belangen des Privatheitsschutzes und der Gewährleistung öffentlicher Sicherheit.

Als Privatsphäre wird ein nichtöffentlicher Bereich bezeichnet, in dem Menschen unbehelligt von äußeren Einflüssen und Kontrollen ihre Persönlichkeit frei entfalten können. ${ }^{36}$ Der Anspruch auf Privatheit gilt als Menschenrecht und seine Gewährleistung als Voraussetzung für das Funktionieren der demokratischen Willensbildung. Dabei setzt die Aufrechterhaltung von Privatheit wiederum informationelle

\section{"Mit dem Übergang zur digitalen Informationsgesellschaft geht eine Verschärfung von Interessenkonflikten einher, weil gegenläufige Positionen zunehmend bedrohlich erscheinen und die Beilegung von Problemen durch Kompromisse immer mehr erschwert wird."}

Das ,Sowohl-als-auch ' wird durch das ,Entweder-oder' als vorherrschendes gesellschaftliches Strukturprinzip abgelöst.

Dies lässt das Verständnis für die Werthaltungen, Lageeinschätzungen und Interessen anderer abnehmen, weil gegenläufige Positionen zunehmend bedrohlich erscheinen, und erschwert selbst Gutwilligen die Verabredung und Umsetzung von Kompromissen. Eben dort ruht aber der Fokus von Konzepten, die auf Kollaboration als „heiligen Gral“34 oder zumindest als „weithin empfohlene Strategie“35 zur Bearbeitung von Wicked Problems setzen.

Damit ist im Übergang zur digitalen Informationsgesellschaft nicht nur mit einer Zunahme von Interessenkonflikten zu rechnen, sondern auch damit, dass sich Zielkonflikte verschärfen bzw. dass sich ihre Beilegung oder Einhegung immer schwieriger gestaltet.

Wie stark Politik und Verwaltung im Übergang zur digitalen Informationsgesellschaft mit widerstreitenden Handlungsimperativen konfrontiert werden, zeigt auch der im Folgenden exemplar-
Selbstbestimmung voraus, welche impliziert, dass den Bürgerinnen und Bürgern auch in einer digitalen Informationsgesellschaft, in der Kommunikationsinhalte von Dritten ausgespäht und Kommunikationsakte umfassend nachvollzogen und zu detaillierten Profilen verdichtet werden können, die Herrschaft über die zu ihrer Person anfallenden Daten zugebilligt wird.

Privatheit und informationelle Selbstbestimmung sind heute aber gleich von zwei Seiten gefährdet, nämlich einerseits durch die Aktivitäten unzureichend kontrollierter Geheimdienste und andererseits durch ihre Marktmacht ausnutzende Internetkonzerne. Politik und Behörden

\footnotetext{
29 Siehe etwa Schulze 2006.

Keilhacker 1968, S. 139.

Hanft/Müskens/Muckel 2004.

BMWi 2016.

Siehe Winkel 2004, S. $10 f$.

AGPSC 2007, S. 27.

Head 2008, S. 114.

Siehe etwa Diggelmann/Cleis 2014, S. 441ff., Heisenberg 2005.
} 
sind daher nicht nur gehalten, das eigene Vorgehen mit dem informationellen Selbstbestimmungsrecht der Gesellschaftsmitglieder in Einklang zu bringen, sondern auch, Maßnahmen zu ergreifen, um es gegen Übergriffe Dritter zu verteidigen.

Als Mittel, um die Ausspähung von sensiblen Informationen und Kommunikationsbeziehungen zu verhindern,
In dem Maße, wie die neuen technischen Potentiale zur Aufdeckung von Kommunikationsbeziehungen, zur Ausforschung von Kommunikationsinhalten und zur Verknüpfung der dabei erzielten Erkenntnisse ausgenutzt werden, droht aber ein Verlust an Privatheit mit fatalen Folgen für Individuum und Gesellschaft, die von der Beeinträchtigung der bürgerlichen Handlungsfreiheit bis zum Verfall

\section{"Der Ausbau der informationstechnischen Infrastruktur, die Förderung von Medienkompetenz und die Austarierung von Belangen von Privatheit und öffentlicher Sicherheit - auch in diesen Feldern zeigt sich, dass Wicked Problems auf dem Vormarsch sind."}

können Anwendungen der elektronischen Kryptographie dienen. ${ }^{37}$ Diese erlaubt neben digitalem Signieren auch eine vertrauliche Inhalte schützende Verschlüsselung und unterstützt Verfahren für anonyme Kommunikation, wie man sie vom Darknet her kennt. ${ }^{38}$

Die Gewährleistung öffentlicher Sicherheit umfasst den Schutz der verfassungsmäßigen Ordnung, des Staates und seiner Einrichtungen und der materiellen und immateriellen Rechtsgüter der Gesellschaftsmitglieder. ${ }^{39}$ Dabei erlangen im Übergang zur digitalen Informationsgesellschaft die Bekämpfung neuer Kriminalitätsformen, man denke etwa an Datendiebstahl, Erpressung mittels Schadsoftware, Anbahnung von Waffenhandel oder Verbreitung von Kinderpornographie ${ }^{40}$, und der Schutz kritischer Infrastrukturen wie Energiewesen oder Verkehrswesen vor terroristischen Übergriffen ${ }^{41}$ zunehmende Bedeutung.

$\mathrm{Zu}$ den Maßnahmen, die sich zum Kampf gegen Internetkriminalität und zur Sicherung kritischer Infrastrukturen eignen, zählen insbesondere die Beobachtung möglicher Gefahrenquellen und die Zusammenführung und Auswertung der dabei gewonnenen Daten. demokratischer Substanz reichen. ${ }^{42}$ Damit offenbart sich an dieser Stelle ein Dilemma: Vieles, was der Aufrechterhaltung der öffentlichen Sicherheit dienen kann, ist unter Aspekten des Privatheitsschutzes bedenklich, und vieles, was zum Schutz von Privatheit geeignet erscheint, ist unter Gesichtspunkten der öffentlichen Sicherheit nicht wünschenswert.

Besonders deutlich wird dies, wenn man die Potentiale in den Blick nimmt, die Anwendungen der elektronischen Kryptographie für den Schutz vertraulicher Inhalte und anonyme Kommunikation bieten. Unter Aspekten des Privatheitsschutzes erscheint der Staat gehalten, die Verbreitung entsprechender Instrumente in der Bevölkerung zu fördern und vielleicht sogar selber eine Infrastruktur zur „Volksverschlüsselung“ und zur anonymen Kommunikation aufzubauen. ${ }^{43}$ Unter Gesichtspunkten der öffentlichen Sicherheit ist aber das Gegenteil der Fall. Denn in einer Gesellschaft, deren Kommunikations- und Kooperationsbeziehungen zunehmend in elektronische Netze verlagert werden, setzen der Schutz kritischer Infrastrukturen und eine effektive Kriminalitätsbekämpfung voraus, dass Sicherheitsbehörden Kenntnis von Kommunikationsbeziehungen haben und gegebenenfalls auch auf deren Inhalte zugreifen können.
Im Übergang vom Analogen zum Digitalen erscheint nicht nur die Entstehung einer totalitären Überwachungsgesellschaft denkbar, sondern ebenso die Herausbildung einer radikalliberalen Anarchie, in der das einzig geltende Recht das des Stärkeren ist. Zunehmend schwierig gestaltet sich aber die Suche nach Wegen, auf denen der Schutz von Privatheit und öffentliche Sicherheit auch unter den veränderten Vorzeichen einer digitalen Informationsgesellschaft in ein ausgewogenes Verhältnis gebracht werden können.

\section{Bedarf an kulturellen und institu- tionellen Innovationen}

Die Herausforderungen, die Politik und Verwaltung im Übergang zur digitalen Informationsgesellschaft aus der Entstehung neuer und der Verschärfung überkommener Wicked Problems erwachsen, sind so gravierend, dass als Antwort darauf auch Maßnahmen in Betracht gezogen werden sollten, die auf grundlegende kulturelle und institutionelle Innovationen abzielen.

Eine Veränderung der Problembearbeitungskultur könnte mit einer umfassenden Sensibilisierung von Funktionsträgern in Politik und Verwaltung für die geschilderten Entwicklungen beginnen. ${ }^{44}$ Das Ziel müsste dabei sein, diese zu befähigen, auch in der Auseinandersetzung mit vermeintlich Altbekanntem übergeordnete Zusammenhänge zu erkennen und dabei neben Konfliktpotential auch mögliche Gemeinsamkeiten mit in anderen Bereichen tätigen und auf anderen Ebenen angesiedelten Akteuren zu identifizieren.

Wenn dies gelänge, könnten vielleicht auch ausgewiesene Verteidiger der Privatheit und eingeschworene Hüter der öffentlichen Sicherheit, deren Auseinan-

\footnotetext{
37 Siehe etwa Spitz/Pramateftakis/Swoboda 2011.

38 Siehe etwa Mey 2017.

39 Siehe etwa Frevel 2018, S. $3 \mathrm{ff}$.

40 Siehe etwa Wernert 2017.

41 Siehe etwa Schulze 2006.

42 Näheres dazu etwa bei Schaal 2004, S. 114 und Winkel 2018, S. $124 \mathrm{ff}$.

43 FISIT 2017.

44 Siehe etwa AGPSC 2007, S. 31ff., Head 2008, S. $107 \mathrm{ff}$. , Weber/Khademian 2008, S. 340f.
} 
dersetzung in der Vergangenheit schon gelegentlich den Charakter eines Rituals angenommen hat, erkennen, dass sie mehr verbindet als auf den ersten Blick ersichtlich. Denn letztlich liegt es auch im Interesse der informationellen Selbstbestimmung, wenn Belange der öffentlichen Sicherheit gewahrt bleiben, und im Interesse der Sicherheitsbehörden, wenn es nicht zum „Ende der Privatheit“45 kommt. Legitimieren sich doch beide Seiten über den Anspruch, Demokratie und Rechtsstaatlichkeit schützen zu wollen. Und sind doch sowohl Demokratie als auch Rechtsstaatlichkeit ohne garantierte individuelle Freiräume ebenso wenig denkbar wie ohne ein System von Regeln und Sanktionsmechanismen, das dem Missbrauch dieser Räume entgegenwirkt. Gelänge es einer Partei, ihren Interessen im Übergang zur digitalen Informationsgesellschaft absolute Geltung zu verschaffen, wäre nicht nur das Projekt der anderen Seite, sondern auch das eigene gescheitert.

In dem Maße, wie sich im Wechsel vom Analogen zum Digitalen der Charakter gesellschaftlicher Probleme verändert und die Interdependenzen zwischen relevanten Faktoren innerhalb und außerhalb der Problemfelder zunehmen, steigt die Notwendigkeit, neben den kulturellen auch die institutionellen Voraussetzungen der Problembearbeitung auf den Prüfstand zu stellen. ${ }^{46}$

Hinsichtlich der Schwierigkeiten, die sich bei der Austarierung von Belangen des Privatheitsschutzes und der öffentlichen Sicherheit ergeben, könnte eine solche Prüfung etwa den Bedarf an neuen Arenen, Gremien oder Foren aufdecken, die Vertreter beider Lager mit Akteuren aus anderen gesellschaftlichen Bereichen zusammenführen und ihnen die Möglichkeit eröffnen, mit wissenschaftlicher Unterstützung Interessen effektiver als zuvor abzugleichen und mit mehr Erfolg als bisher auf mehrseitig akzeptable Lösungen hinzuarbeiten.

Dabei sind durchaus Anknüpfungspunkte für die Annäherung von Datenschützern und Sicherheitspolitikern in Sicht. So beeinträchtigen massenhaft Nutzerdaten sammelnde und diese zu detaillierten Profilen verdichtende Internetkonzerne nicht nur die informationelle Selbst- bestimmung der Gesellschaftsmitglieder. Ihre Aktivitäten laufen auch der Aufgabe von Sicherheitsbehörden zuwider, Bürgerinnen und Bürger gegen Übergriffe Dritter zu schützen. Hinzu kommt, dass die dadurch im privatwirtschaftlichen Sektor entstehende Macht auch in einer das politische Gemeinwesen unmittelbar gefährdenden Weise eingesetzt werden kann, wie

\section{Schluss}

Die Digitalisierung führt zur Zunahme von sozialer Komplexität, Diversität und Unsicherheit. Außerdem leistet sie einer gravierenden Zuspitzung von Zielkonflikten Vorschub. Daher ist im Übergang zur digitalen Informationsgesellschaft mit der Entstehung neuer und der Verschär-

\section{"Zur Anpassung der gesellschaftlichen Problembearbeitungsfähigkeit an die gestiegenen Anforderungen sind kulturelle und institutionelle Innovationen erforderlich."}

etwa der Fall Cambridge Analytica zeigt. ${ }^{47}$

Wenn neue Institutionen zur Verfügung stehen, sollten diese genutzt werden, um möglichst einfach gehaltene Lösungsstrategien zu entwickeln. Der auf den ersten Blick naheliegende Gedanke, jedes Wicked Problem bedürfe einer „Wicked Solution “48, führt in die Irre. Denn wenn die Strategie zur Bearbeitung eines komplexen Problems ebenso komplex ist wie das Problem selbst, liegt angesichts des daraus resultierenden Komplexitätszuwachses die Vermutung nahe, dass sie die Lage eher verschärfen als entschärfen wird.

So könnte man dem Misstand, dass es bei Konzernen wie Alphabet oder Facebook an Transparenz und demokratischer Kontrolle fehlt, obwohl deren Wissen die Erkenntnisse von Geheimdiensten und deren Umsatz das Bruttosozialprodukt von Staaten übertrifft, zuerst einmal durch die Forderung nach einem verbesserten Schutz von Whistleblowern aus der Internetwirtschaft begegnen. Dies wäre weitaus schneller und leichter $\mathrm{zu}$ realisieren als etwa die Etablierung einer grenzüberschreitend agierenden „European Data Agency“, die dafür sorgt, dass „alle Firmen, die mit unseren Daten operieren, jederzeit und vollständig nach unseren Regeln spielen “. 49 fung überkommener Wicked Problems zu rechnen. Dies stellt Politik, Verwaltung und andere mit der Erfüllung öffentlicher Aufgaben befasste Akteure vor große Herausforderungen. Zur Anpassung der gesellschaftlichen Problembearbeitungsfähigkeit an die gestiegenen Anforderungen sind kulturelle und institutionelle Innovationen erforderlich. 


\section{Literatur}

Australian Government Public Service Commission AGPSC, 2007: Tackling wicked problems, Canberra: Verlag der AGPSC.

Banse, Gerhard, 1996: Herkunft und Anspruch der Risikoforschung, in: Banse, Gerhard (Hrsg.): Risikoforschung zwischen Disziplinarität und Interdisziplinarität. Von der Illusion der Sicherheit zum Umgang mit Unsicherheit, Berlin: Edition Sigma, S. 15-72.

Beck, Ulrich, 1998: Was ist Globalisierung?, Frankfurt am Main: Suhrkamp.

Bleicher, Joan, 2010: Internet, Konstanz: UVK Verlagsgesellschaft.

Brugnach, Marcela/Ingram, Helen, 2017: Ways of knowing and relational knowledge, in: Weber, Edward/Lach, Denise/Steel, Brent (Hrsg.): New strategies for wicked problems, Corvallis: Oregon State University Press, S. 27-43.

Bundesministerium für Wirtschaft und Energie BMWi, 2016: Digitale Bildung. Der Schlüssel zu einer Welt im Wandel, Berlin: Verlag des BMWi.

Cheng, Tsuo-Yu, 2012: Soziale Geschwindigkeit. Ein theoretischer Grundriss und eine zeitpolitische Fragestellung, Berlin: Verlag der FU Berlin.

Churchman, Charles, 1967: Wicked problems, in: Management Science, 4, S. 141-142.

Diggelmann, Oliver/Cleis, Nicole, 2014: How the right to privacy became a human right, in: Humans Rights Law Review, 14, S. 441-458.

Fischbacher-Smith, Denis, 2016: Framing the UK's counter-terrorism policy within the context of a wicked problem, in: Public Money and Management, 6, S. 399-408.

Fraunhofer Institut für sichere Informationstechnologie FISIT, 2017: Projekt Volksverschlüsselung, Darmstadt: Verlag des FISIT.

Frevel, Bernhard, 2018: Innere Sicherheit. Eine Einführung, Wiesbaden: Springer.

Fuchs-Heinritz, Werner/Lautmann, Rüdiger/ Rammstedt, Otthein/Wienold, Hanns, 1995: Lexikon zur Soziologie, Opladen: Westdeutscher Verlag.

Goldsmith, Stephen/Eggers, William, 2004: Governing by network, Washington DC: Brookings Institution.

Hanft, Anke/Müskens,Wolfgang/Muckel, Petra, 2004: Zertifizierung und Nachweis von ITKompetenzen, Oldenburg: Verlag der Ossietzky Universität.

Head, Brian, 2008: Wicked problems in public policy, in: Public Policy, 2, S. 101-118.

Head, Brian/Alford, John, 2015: Wicked problems - implications for public policy and management, in: Administration and Society, 6, S. 711739.

Heisenberg, Dorothee, 2005: Negotiating privacy. The European Union, the United States, and personal data protection, Boulder in Colorado: Lynne Rienner Publishers.

Hirsch-Kreinsen, Hartmut, 2016: Zum Verhältnis von Arbeit und Technik bei Industrie 4.0, in: Aus Politik und Zeitgeschichte, 18-19, S. 10-17.

Horn, Robert/Weber, Robert, 2007: New tools for resolving wicked problems. Mess mapping and resolution mapping processes, Stanford: Verlag der Stanford University.
Hulme, Mike, 2009: Why we disagree about climate change. Understanding controversy, inaction and opportunity, Cambridge: Cambridge University Press.

Kaiser, Brittany, 2019: Targeted. My inside story of Cambridge Analytica, New York: Harper Collins Publishers.

Keilhacker, Martin, 1968: Der Mensch von heute in der Welt der Informationen, in: Jugend Film Fernsehen, 3, S. 131-146.

Lessig, Lawrence, 1997: The constitution of code, in: CommLaw Conspectus, 5, S. 181-193.

Levin, Kelly/Cashore, Benjamin/Bernstein, Steven/Graeme, Auld, 2012: Overcoming the tragedy of super wicked problems, in: Policy Sciences, 4, S. 123-152.

Mai, Manfred, 2007: Die Informationsgesellschaft als Utopie und Versprechen, in: Die Politische Meinung, 5, S. 14-20.

Martini, Mario, 2016: Digitalisierung als Herausforderung und Chance für Staat und Verwaltung, Speyer: Verlag des Deutschen Forschungsinstituts für öffentliche Verwaltung Speyer.

Mey, Stefan, 2017: Darknet - Waffen, Drogen, Whistleblower, München: Beck.

Müller-Wirth, Moritz/Wefing, Heinrich, 2016: Ich, in: Die Zeit Nr. 46 vom 3. November, S. 4.

Organization for Economic Cooperation and Development OECD, 2019: Going digital, Paris: Verlag der OECD.

Ritchey, Tom, 2013: Wicked Problems. Modelling social messes with morphological analysis, in: Acta Morphologica Generalis, 1, S. 1-8.

Rittel, Horst/Webber, Melvin, 1973: Dilemmas in a general theory of planning, in: Policy Sciences, 4, S. 155-169.

Rosa, Hartmut, 2005: Beschleunigung. Die Veränderung der Zeitstrukturen in der Moderne, Frankfurt am Main: Suhrkamp.

Schaal, Gary, 2004: Die Zukunft der Demokratie, in: Reder, Michael/Cojocaru, Mara-Daria (Hrsg.): Zukunft der Demokratie - Ende einer Illusion oder Aufbruch zu neuen Formen?, Stuttgart: Kohlhammer, S. 109-124.

Schulze, Tillmann, 2006: Bedingt abwehrbereit. Schutz kritischer Informationsinfrastrukturen in Deutschland und den USA, Wiesbaden: VS.

Schumacher, Ernst, 1995: A guide for the perplexed, London: Vintage.

Schwarz-Wölzl, Maria/Maad, Christa, 2004: Diversity und Managing Diversity. Teil 1: Theoretische Grundlagen, Wien: Verlag des Zentrums für soziale Innovation.

Siegele, Ludwig, 2019: Das Huawei-Dilemma, in: Die Zeit Nr. 11 vom 7. März, S. 23.

Spicker, Paul, 2016: Poverty as a wicked problem, in: Poverty Brief, 3, S. 1-4.

Spitz, Stephan/Pramateftakis, Michael/ Swoboda, Joachim, 2011: Kryptographie und ITSicherheit, Wiesbaden: Vieweg.

Vogel, Johannes, 2019: Spielregeln für Giganten, in: Die Zeit Nr. 31 vom 25. Juli, S. 6.

Watkins, Alan/Wilber, Ken, 2015: Wicked and wise, Kent: Urbane Publications.

Weber, Edward/Khademian, Anne, 2008: icked problems, knowledge challenges, and collaborative capacity builders in network settings, in: Public Administration Review, 2, S. 334-349.
Weber, Edward/Lach, Denise/Steel, Brent, 2017: Science and problem solving for wicked problems, in: Weber, Edward/Lach, Denise/Steel, Brent (Hrsg.): New strategies for wicked problems, Corvallis: Oregon State University Press, S. 1-24.

Weigand, Florian/Bieker, Lisa/Gorny, Dominic/Weber, Mike, 2015: ÖFIT-Atlas der Digitalisierung, Berlin: Verlag des Kompetenzzentrums Öffentliche IT.

Wernert, Manfred, 2017: Internetkriminalität, Stuttgart: Boorberg.

Whitaker, Reg, 2000: The end of privacy. How total surveillance is becoming a reality, New York: New Press.

Winkel, Olaf, 2004: Zukunftsperspektive Electronic Government, in: Aus Politik und Zeitgeschichte, 18, S. 7-15.

Winkel, Olaf, 2018: Entwicklungslinien, Handlungsfelder und widerstreitende Handlungsimperative der Digitalisierung in Staat und Verwaltung, in: Verwaltung und Management, 3, S. 115-130.

Wolf, Thomas/Strohchen, Jacqueline-Helena, 2018: Digitalisierung - Definition und Reife, in: Informatik-Spektrum, 1, S. 56-64. 